\title{
A BOUND FOR DECOMPOSITIONS OF $m$-CONVEX SETS WHOSE LNC POINTS LIE IN A HYPERPLANE ${ }^{1}$
}

\author{
MARILYN BREEN
}

\begin{abstract}
A set $S$ in $R^{d}$ is said to be $m$-convex, $m>2$, if and only if for every $m$ points in $S$, at least one of the line segments determined by these points lies in $S$. Let $S$ denote a closed $m$-convex set in $R^{d}$, and assume that the set of lnc points of $S$ lies in a hyperplane. Then $S$ is a union of $f(m)$ or fewer convex sets, where $f$ is defined inductively as follows: $f(2)=1$, $f(3)=2$, and $f(m)=f(m-2)+3$ for $m \geqslant 4$. Moreover, for $d>3$, an example reveals that the best bound is no lower than $g(m)$, where $g(m)=$ $f(m)$ for $2<m<5$ and for $m=7$, and $g(m)=g(m-3)+4$ otherwise.
\end{abstract}

1. Introduction. Let $S$ be a subset of $R^{d}$. The set $S$ is said to be $m$-convex, $m \geqslant 2$, if and only if for every $m$ distinct points in $S$, at least one of the line segments determined by these points lies in $S$. A point $x$ in $S$ is said to be a point of local convexity of $S$ if and only if there is some neighborhood $N$ of $x$ such that $N \cap S$ is convex. If $S$ fails to be locally convex at some point $q$ in $S$, then $q$ is called a point of local nonconvexity (lnc point) of $S$. As in [1], the following terminology will be used: For $x, y$ in $S$, we say $x$ sees $y$ via $S$ if and only if the corresponding segment $[x, y]$ lies in $S$. Points $x_{1}, \ldots, x_{n}$ in $S$ are visually independent via $S$ if and only if for $1 \leqslant i<j \leqslant n, x_{i}$ does not see $x_{j}$ via $S$. For $x$ in $S$, the set of all points of $S$ which see $x$ via $S$ is called the star of $x$ in $S$, denoted $S_{x}$. Throughout the paper, cl $S$, int $S$, and rel int $S$ will be used to denote the closure, interior, and relative interior, respectively, of the set $S$.

Several decomposition theorems have been obtained for closed $m$-convex sets $S$ in the plane [4], [3], [2], and if we further require the set of lnc points of $S$ to lie on a line, then $S$ will be a union of $m-1$ or fewer convex sets [1]. Here we investigate an analogous problem in $R^{d}, d \geqslant 3$, obtaining a decomposition theorem for closed $m$-convex sets in $R^{d}$ whose lnc points lie in a hyperplane. Moreover, an example reveals that for $d \geqslant 3$ and $m \geqslant 4$, the planar bound $m-1$ is no longer possible.

\section{The results.}

THEOREM 1. Let $S=\operatorname{cl}\left(\right.$ int $S$ ) be an $m$-convex set in $R^{d}, m \geqslant 2$, and assume that the set $Q$ of lnc points of $S$ lies in a hyperplane $H$. Then $S$ is a union of $f(m)$ or fewer convex sets, where the function $f$ is defined inductively as follows:

Received by the editors December 5, 1977.

AMS (MOS) subject classifications (1970). Primary 52A20, 52A40.

'This research was sponsored in part by an Arts and Sciences Summer Fellowship at the University of Oklahoma. 
$f(2)=1, f(3)=2$, and $f(m)=f(m-2)+3$ for $m \geqslant 4$.

Proof. We use an inductive argument. The result is trivial for $m=2$, and if $m=3$, it follows immediately from [1, Theorem 1]. Hence suppose that $m \geqslant 4$ and assume that the theorem is true for all integers $k, 2 \leqslant k<m$, to prove for $m$.

Let $H_{1}$ and $H_{2}$ denote distinct open halfspaces determined by the hyperplane $H$. By the $m$-convexity of $S$, it is easy to see that for $i=1,2$, each set $S \cap H_{i}$ has at most $m-1$ components, each starshaped. Furthermore, standard arguments involving a result by Valentine [5, Corollary 1] reveal that each of these components is convex. Since $S=\operatorname{cl}($ int $S$ ), every point of $S$ must lie in the closure of at least one of these components.

We show that, without loss of generality, we may assume every point of $S \cap H$ lies in $\operatorname{cl}\left(S \cap H_{1}\right) \cap \operatorname{cl}\left(S \cap H_{2}\right)$ : Otherwise, for some point $x$ in $S \cap H$ and for an appropriate labeling of the halfspaces $H_{1}$ and $H_{2}, x \notin \operatorname{cl}(S$ $\left.\cap H_{1}\right)$. Then since $S=\operatorname{cl}($ int $S), x \in \operatorname{cl}\left(S \cap H_{2}\right)$. By previous comments, for some $k, 1 \leqslant k \leqslant m-1, x$ is in the closure of exactly $k$ components of $S \cap H_{2}$, and if we choose one point from each of these components, clearly these points will be visually independent via $S$. If $k=m-1$, then it is easy to show that $S_{x}=S, S=\operatorname{cl}\left(S \cap H_{2}\right)$, and $S$ is a union of $m-1<f(m)$ or fewer convex sets. If $k \leqslant m-2$, then a proof similar to [1, Lemma 1] may be used to show that the set $S^{\prime} \equiv \operatorname{cl}\left(S \sim S_{x}\right)$ is at most $(m-k)$-convex, $S^{\prime}=\operatorname{cl}\left(\right.$ int $S^{\prime}$ ), and the lnc points of $S^{\prime}$ are in $Q$. Hence by our induction hypothesis, $S^{\prime}$ is a union of $f(m-k)$ or fewer convex sets. It is not hard to show that cl(int $\left.S_{x}\right)$ is a union of $k$ convex sets, and we conclude that

$$
S=\operatorname{cl}\left(\text { int } S_{x}\right) \cup \operatorname{cl}\left(S \sim S_{x}\right)
$$

is a union of $f(m-k)+k \leqslant f(m)$ or fewer convex sets, the desired result. Hence throughout the remainder of the proof we may assume that

$$
S \cap H \subseteq \operatorname{cl}\left(S \cap H_{1}\right) \cap \operatorname{cl}\left(S \cap H_{2}\right) .
$$

Similarly, we may assume that for every component $C$ of $S \sim H$, cl $C \cap H$ is a full $(d-1)$-dimensional: Otherwise, for some component $C$ of $S \sim H, S$ will be the union of the convex set $C$, and the $(m-1)$-convex set $\operatorname{cl}(S \sim C)$, giving a decomposition of $S$ into $1+f(m-1) \leqslant f(m)$ of fewer convex sets, again the appropriate bound.

To complete the proof, we consider two cases:

Case 1. Assume for the moment that there exist some lnc point $q$ of $S$ and some component $C$ of $S \sim H$ such that $q \in \operatorname{rel} \operatorname{int}(\mathrm{cl} C \cap H)$. (Recall that cl $C \cap H$ is $(d-1)$-dimensional.) For convenience of notation, let $C \subseteq H_{1}$. Then $q$ is in the closure of $k$ components of $S \cap H_{2}$. Moreover, since we are assuming that

$$
S \cap H \subseteq \operatorname{cl}\left(S \cap H_{1}\right) \cap \operatorname{cl}\left(S \cap H_{2}\right),
$$

it is clear that $2 \leqslant k$. Hence $2 \leqslant k \leqslant m-1$. It is easy to see that the set 
cl(int $S_{q}$ ) is a union of $k+1$ convex sets, each the closure of a component of $S \sim H$. If $k=m-1$, then standard arguments show that $S=S_{q}$, and $S$ is a union of $k+1=m \leqslant f(m)$ or fewer convex sets. If $k \leqslant m-2$, then again the proof of [1, Lemma 1] reveals that the $\operatorname{set} \operatorname{cl}\left(S \sim S_{q}\right)$ is at most ( $m-$ $k$ )-convex and satisfies our induction hypothesis. Thus $\operatorname{cl}\left(S \sim S_{q}\right)$ is a union of $f(m-k)$ or fewer convex sets, and $S$ is a union of $f(m-k)+k+1$ convex sets. But for $2 \leqslant k \leqslant m-2$,

$$
f(m-k)+k-2 \leqslant f(m-k+k-2)=f(m-2),
$$

so $f(m-k)+k+1 \leqslant f(m-2)+3=f(m)$, and $S$ is a union of $f(m)$ or fewer convex sets, finishing the argument for Case 1.

Case 2. If Case 1 does not occur, then select any component $C$ of $S \cap H_{1}$. By previous assumptions, there exists a component $D$ of $S \cap \mathrm{H}_{2}$ such that

$$
\text { rel int }(\operatorname{cl} C \cap H) \cap \operatorname{rel} \operatorname{int}(\operatorname{cl} D \cap H) \neq \varnothing .
$$

Moreover, $D$ must be unique: Otherwise, for appropriate components $D_{1}$ and $D_{2}$ of $S \cap H_{2}$, cl $D_{1} \cap \mathrm{cl} D_{2} \cap \operatorname{rel} \operatorname{int}(\operatorname{cl} C \cap H) \neq \varnothing$, and there would be some lnc point of $S$ in rel int(cl $C \cap H$ ), contradicting our hypothesis for Case 2. Similarly, $C$ is the unique component of $S \cap H_{1}$ for which rel int(cl $C \cap H) \cap \operatorname{rel} \operatorname{int}(\operatorname{cl} D \cap H) \neq \varnothing$, and $\operatorname{cl} C \cap H=\operatorname{cl} D \cap H$. Note that the set $\operatorname{cl} C \cup \operatorname{cl} D$ is $j$-convex, where $j$ is either 2 or 3 . Now since $S=\operatorname{cl}($ int $S$ ) and $\operatorname{cl} C \cap H=\operatorname{cl} D \cap H$, it is easy to show that for any point $z$ in $\operatorname{cl} C \cup \operatorname{cl} D$ and for any $w$ in $S \sim(\operatorname{cl} C \cup \operatorname{cl} D)$, there is a sequence $\left\{w_{n}\right\}$ in $S$ converging to $w$ such that no $w_{n}$ sees $z$ via $S$. Hence standard arguments similar to those in [1, Lemma 1] may be used to show that the $\operatorname{set} \operatorname{cl}(S \sim(\operatorname{cl} C \cup \operatorname{cl} D))$ is $(m-j+1)$-convex and satisfies our induction hypothesis. We conclude that $S$ is a union of $(j-1)+f(m-j+$ $1) \leqslant f(m)$ or fewer convex sets. This completes Case 2 and finishes the proof of the theorem.

It is interesting to note that for $d \geqslant 3$, without the requirement that $S=\operatorname{cl}($ int $S$ ), the theorem fails and, in fact, no finite decomposition is possible. (See [1, Remarks 1 and 2].)

In conclusion, the following example reveals that for $d \geqslant 3$, the best bound is no lower than $g(m)$, where $g(m)=f(m)$ for $2 \leqslant m \leqslant 5$ and for $m=7$, and $g(m)=g(m-3)+4$ otherwise.

EXAMPLE 1. Let $g$ be a function defined inductively as follows: $g(2)=1$, $g(3)=2, g(4)=4$, and $g(m)=g(m-3)+4$ for $m \geqslant 5$. Then for each $m \geqslant 2$ and $d \geqslant 3$, there corresponds an $m$-convex set $T_{m, d}$ in $R^{d}$ which satisfies the hypothesis of Theorem 1 and which is expressible as a union of $g(m)$ and no fewer convex sets.

Examples for $m=2$ and $m=3$ are trivial. Hence let $m=4$ and, for convenience of notation, let $d=3$. Define $x_{1}=(2,0,0), x_{2}=(0,2,0), x_{3}=$ $(-2,0,0), x_{4}=(0,-2,0)$. Define $R_{i} \equiv \operatorname{conv}\left\{x_{j}: j \neq i\right\}, 1 \leqslant i \leqslant 4$, and let $y_{1}=(-1,0,1), y_{3}=(1,0,1), y_{2}=(0,-1,-1), y_{4}=(0,1,-1)$. Note that, 
looking down on the $x-y$ plane from the positive $z$ axis, $y_{i}$ is above the corresponding $R_{i}$ set for $i=1,3$, below for $i=2$, 4.) Finally, let

$$
T_{4,3}=\cup\left\{\operatorname{conv}\left(R_{i} \cup\left\{y_{i}\right\}\right): 1 \leqslant i \leqslant 4\right\} .
$$

Then $T_{4,3}$ is a 4-convex set which is a union of 4 and no fewer convex sets. This example may be generalized to higher dimensions to construct $T_{4, d}$.

Inductively, for $m \geqslant 5$, let $T_{m, d}$ be the disjoint union of $T_{m-3, d}$ and $T_{4, d}$. Then $T_{m, d}$ will be an $m$-convex set in $R^{d}$ expressible as a union of $g(m-3)$ $+4=g(m)$ and no fewer convex sets. Hence the planar bound of $m-1$ fails when $d \geqslant 3$ and $m \geqslant 4$.

\section{REFERENCES}

1. Marilyn Breen, M-convex sets whose lnc points lie in a hyperplane, J. London Math. Soc. 16 (1977), 529-535.

2. Marilyn Breen and David C. Kay, General decomposition theorems for $m$-convex sets in the plane, Israel J. Math. 24 (1976), 217-233.

3. H. G. Eggleston, A condition for a compact plane set to be a union of finitely many convex sets, Proc. Cambridge Philos. Soc. 76 (1974), 61-66.

4. F. A. Valentine, $A$ three point convexity property, Pacific J. Math. 7 (1957), 1227-1235.

5. __ Local convexity and $L_{n}$ sets, Proc. Amer. Math. Soc. 16 (1965), 1305-1310.

Department of Mathematics, University of OxLahoma, Norman, Oxiahoma 73019 